\title{
Zur Kritik der strikten Anwendung des Prioritätsprinzips in der Nomenklatur.
}

\author{
Der Gattungsname Dibotia.
}

Von Franz Heikertinger, Wien.

In den letzten Jahren ist die Klage über die unhaltbaren Verhältnisse in der Nomenklatur der Lebewesen immer lauter geworden. Während aber der eine Forscher das trostlose Bild der nomenklatorischen Gegenwart nur für ein Übergangsstadium hält und das alleinige Heil im eisernen „Durchhalten“ des starren Prioritätsprinzips sieht, glaubt der andere in eben demselben starren Prinzipe die Wurzel alles Übels zu erblicken und fordert dessen Durchbrechung.

Es sei mir gestattet, mit dem Folgenden einen kleinen Beitrag zur Klärung der Frage zu geben. Eingedenk des. Satzes, das Beispiel wirke mehr als das Wort, übergebe ich dieses Exempel den verehrten Kollegen mit der Bitte, es vorurteilslos zu prüfen.

Nahezu ein Jahrhundert lang gilt für jene Gattung der einheimischen Erdflöhe, die sich durch einen gegabelten Hinterschienendorn vor allen andern aufällig kennzeichnet, der Name Dibotia. Dieser Name ist nie durch einen anderen bedrängt worden: seit seinem Aufkommen, seit der Aufstellung der Gattung als solcher ist er deren einzige, einheitliche, allgemein bekannte Bezeichnung. Wenn wirklich Stabilität der Endzweck aller Nomenklaturregeln ist, wie allseits versichert wird und wie es logisch auch sein mup, dann mufo zugegeben werden, daß die Nomenklaturregeln nichts anderes zu tun haben können, als diesen Namen mit allen Mitteln zu erhalten. Denn dieser Name verkörpert eine fertige, einwandfreie, eingelebte, jahrhundertalte Stabilität, einen Begriff, der jedem Coleopterologen geläufig ist und der unter diesem Namen tausendfach in die systematische und biologische Literatur eingeflochten ist.

Wenn wir dem Namen Dibolia den Namen eines Autors anhängen, so geschieht dies eigentlich mehr aus Gewohnheit als aus irgend einem vernunftgemäßen Grunde. Der immer eindeutig gewesene, längst Gemeingut der Coleopterologen gewordene Name Dibolia wird durch ein angefügtes „Latr." oder „Steph." in keiner Weise 
wirksam ergänzt; auch ohne „Latr.“ oder „Steph.“ denkt niemand an etwas anderes als an das, was fast ein Jahrhundert lang in der gesamten wissenschaftlichen Literatur wie in jedem Käferbuche für Jungen "Dibolia" hieß. Das angefügte Autorzitat hätte lediglich für eine allgemein zoologische Nomenklatur den einzigen Wert, vor eventuellen Homonymen zu bewahren. Vor der Verwirrung nämlich, die entstehen könnte, wenn ein Forscher heute einen Eingeweidewurm oder einen niederen Kruster als "Dibolia" benannte, worauf man allerdings nicht mehr wüßte, ob der Erdfloh Dibolia oder der Eingeweidewurm Dibolia gemeint sei und solches dann allein aus dem Autornamen zu erkennen vermöchte.

Ob dieser Grund hinreicht, dem Namen der Halticine Dibolia immer und überall das schleppende Bleigewicht eines Autornamens anzuhängen, mögen Berufene entscheiden. Wir sind der Meinung, daß ein heutiger Forscher, der den Namen Dibolia neu verwendet, sich eines groben Verstoßes gegen seine Verpflichtungen schuldig macht und daß wir auf einen solchen Fall überhaupt nicht Rücksicht zu nehmen brauchen. Ehe ein Forscher einen neuen Gattungsnamen verleiht, muf er doch wohl einen Blick in die derzeit vorhandenen "Nomenklatoren" (Scudder, Waterhouse, Zoological Record) werfen. Verwendet er einen erst in allerletzter Zeit gegebenen Namen nochmals, so mag ihm das Bereitsvorhandensein dieses Namens entgangen sein; vielleicht fehlte ihm die Möglichkeit, in die allerneueste Literatur Einblick zu nehmen. Verwendet er jedoch einen Namen, den der älteste Nomenklator des vorigen Jahrhunderts schon ausweist, noch einmal, so fehlt die Entschuldigung hiefür. Übrigens würde eine derartige Homonymie, falls sie wider alles Erwarten doch einmal geschaffen werden sollte, bald festgestellt und entfernt sein.

Die Mitschleppung eines Autornamens bei Dibolia ist also praktisch überflüssig.

Erachtet man sie indes an irgend einer Stelle einmal nicht für überflüssig, hält man den Homonymenschutz in irgend einem möglichen Falle doch für wesentlich, so ist es im Grunde ohne Belang, ob wir „Dibolia Latr.", „Dibolia Steph." oder „Dibolia Weise" sagen. Alle drei weisen ja auf eine und dieselbe Gattung hin und darauf kommt es doch bei einem Homonymenschutz allein an. Gezänk über eine Priorität im Autornamen fällt also sicherlich ins Kleinliche.

Der die Wirklichkeit klar durchblickende Forscher wird feststellen : 
Der alte zoologische Name Dibolia bedarf keines Homonymenschutzes, keines angefügten Autornamens. In allgemein wissenschaftlichen Werken kann ihm zur Sicherung gegen - ganz unwahrscheinliche - Homonymien der Name eines Schriftstellers angefügt werden, bei dem wir eine Charakteristik der gemeinten Gattung finden. Praktisch am wertvollsten in jeder Hinsicht wird unleugbar der Name eines solchen Schriftstellers sein, bei dem wir eine brauchb.are, zeitgemäße Charakteristik der Gattung finden.

Das wäre der Standpunkt der Praxis.

Die Nomenklaturregeln, die vielfach mehr mit theoretischen Möglichkeiten als mit den lebendigen Forderungen des wissenschaftlichen Alltags gerechnet haben, bringen ein anderes, mehr oder minder weltfremdes Element in die Angelegenheit. Sie vertreten, wie jedes erstarrende Gesetz, im wesentlichen das Regieren mit Buchstaben.

Sie fordern beispielsweise das Prioritätsprinzip auch für den Autornamen. Wenn die Nennung eines Autors wünschenswert sei, so - verlangen sie - müsse derjenige Schriftsteller genannt werden, der als Erster den Tiernamen in Begleitung einer Kennzeichnung veröffentlichte. Sie wollen die Quelle sichern.

Nun wird es immer und überall wohlmeinende Allzueifrige geben, die, ohne Arges zu denken, die Form über den Inhalt stellen; die unbewuft und in lauterster Absicht den lebendigen Geist mit dem toten Buchstaben erschlagen. Die belanglose Nebendinge als Hauptsachen behandeln, die den Endzweck des Ganzen hiebei allmählich aus den Gesichtskreis verlieren und ihm schließlich unbewußt entgegenwirken.

Dieses Stadium ist heute der Nomenklaturfrage beschieden. Ein Symptom hiefür ist die Überwertung des - wie wir gesehen haben - praktisch recht bedeutungslosen Autornamens und die verbreitete Meinung, die strenge Durchführung des Prioritätsprinzips im Autornamen sei ebenso wichtig wie die strenge Durchführung dieses Prinzips im Tiernamen.

Es ergaben sich darum auch - um zur Gattung Dibolia zurückzukommen - Meinungsverschiedenheiten darüber, welcher von all den belanglosen Autornamen dem Gattungsnamen Dibolia angehängt werden müsse und welcher von allen der allerälteste - also auch der wertloseste, denn je älter die Beschreibung, desto schlechter, unschärfer ist sie in der Regel - sei. Nur der allerälteste, allerwertloseste darf bestehen. 
Bedel${ }^{1}$ ) war es vergönnt, zu entdecken, daß es nicht „Dibolia Latr.", sondern "Dibolia Steph." heißen müsse. Er stellte fest:

„Dibolia Steph., 1831. - Syn. Haltica (8e groupe) Ill.; Galeruca (subg. Dibolia) Latr., 1829."

Diese Feststellung nun ist in zweifacher Beziehung falsch. Erstens ist „Dibolia Latr., 1829“ ein nomenklatorisch völlig einwandfreier, gültiger Name und hat unbedingt die Priorität vor „Dibolia Steph., 1831". Die Synonymie, die Bedel gibt, ist ein logischer Widerspruch. Bedel muß mit unverständlicher Flüchtigkeit Latreilles nomenklatorisch ungültige Erstarbeit, ${ }^{2}$ ) die im Jahre 1825 erschien, mit dessen nomenklatorisch gültiger zweiter Arbeit, ${ }^{3}$ ) die im Jahre 1829 publiziert wurde, irgendwie verwechselt haben.

Aber wenn auch Latreilles zweite Arbeit ebenso wie seine erste nomenklatorisch ungültig wäre, brauchte es immer noch nicht "Dibolia Steph." zu heißen. Denn vor Stephens Arbeit und vor Latreilles zweiter Arbeit wurde durch Berthold d) eine deutsche Übersetzung von Latreilles erster Arbeit publiziert, die 1827 erschien und die an Stelle Latreilles französischer - also nomenklatorisch ungültiger - Namen korrekte latinisierte Tiernamen bringt, also nomenklatorisch gültig ist. Es könnte also vielleicht - wir nehmen an, wir hätten noch nicht nachgesehen - auch heißen: „Dibolia Berth., 1827".

Was allerdings damit gewonnen sein sollte, wenn statt des genialen Latreille, des bedeutendsten Insektenkenners seiner Zeit und eines der größten Bahnbrecher der Entomologie, nunmehr sein - Translator, der bei der Übersetzung der toten Worte vielleicht überhaupt kein Bild dieser Tiere im Geiste hatte, als "Autor" fungierte, das vermag ein unbefangen Denkender nicht zu erfassen. Ich habe an anderer Stelle ${ }^{5}$ ) für diese Fälle Latreille-Bertholdscher Gattungsnamen die Zitatform: „Berth. (ex. Latr.)“ vorgeschlagen, die wenigstens teilweise die geistige Urheberschaft Latreille $\mathrm{s}_{\mathrm{i}}^{\mathrm{z}} \mathrm{um}$ Ausdruck bringt.

Besonderen Wert möchte ich aber auch dieser Zitatform auf keinen Fall beimessen. Fürs erste darum nicht, weil ich, wie erwähnt, das Autorzitat und speziell das Zitat alter Autoren an sich für
1) L. Be d el, Faune d. Coléopt. d. Bassin d. l. Seine. V. 197.
2) M. Latreille, Familles naturelles du Règne animal. Paris, 1825. 405.
$\left.{ }^{3}\right)$ M. Latreille (Cuvier), Le Règne animal. V. Paris, 1829, 154.
${ }^{4}$ ) Latreilles Natürliche Familien des Tierreichs. A. d. Franz. v. Dr.

A. A. Berthold, Weimar, 1827, 401.

5) Entomol. Blätter, X, 266, 1914.

Wiener Entomologische Zeitung, XXXV. Jahrg., Heft III-IV (25. April 1916). 
geringwertig halte, und fürs zweite nicht, weil ich der Anschauung bin, daß ein Autorzitat als Hinweis auf eine brauchbare Charakteristik des Tieres, nicht aber als ein Denkmal für den Erstbeschreiber aufzufassen sei. Gerade die letztere unselige Auffassung des Autornamens hat die für jede ernst wissenschaftliche Bestrebung so verderbliche sogenannte „Mihi-Sucht" der Amateure großgezogen. Das Arten- und "Varietäten"-Beschreiben ist zur grotesken Unsterblichkeitsmanie geworden.

Uns soll es gleich gelten, mit welchem Schriftstellernamen der einzige Zweck einer solchen Autornennung, nämlich die Unterscheidung von Homonymen, erreicht wird. Daß hiefür jeder Name eines Schriftstellers, bei dem wir eine klare Charakteristik finden, geeignet ist, steht außer Frage. Daß anderseits der Name des Erstbeschreibers für jeden anderen Zweck unbrauchbar ist, wird keinem Forscher, der Wirklichkeitsblick besitzt, verborgen geblieben sein.

Als Quellennachweis ist er wertlos, denn aus dieser Quelle könnten wir nichts schöpfen als eine Gefährdung heute gültiger Namen.

Ein Tiername kann von jedem Schriftsteller anders gedeutet worden sein - nichtsdestoweniger wird jeder dieser Schriftsteller dem Tiernamen eines und dasselbe nichtssagende, schablonenhafte "L." oder "Latr." oder „Steph." angehängt haben. Wie sollte dieses „L." oder „Latr." oder „Steph." nun zum Ausdrucke bringen, mit der Deutung welches Schriftstellers wir es in einem gegebenen Falle zu tun haben, welches Tier also eigentlich gemeint sei? Dies könnte doch wohl nur erreicht werden, wenn wir den Schriftsteller nennen, dessen Deutung wir im gegebenen Falle gefolgt sind, nach dessen Auffassung wir uns bei Bestimmung des vorliegenden Tieres wirklich gerichtet haben.

Der schablonenhaft angehängte Name des Erstbeschreibers schützt uns vor Homonymien, ist jedoch aufer stande, die gemeinte Tierform klar und eindeutig zu bezeichnen, ist daher letzten Endes wertlos.

Der Name eines modernen Schriftstellers hingegen schützt uns vor Homonymien und sagt uns überdies klar und unvermittelt, mit welcher Auffassung des Tiernamens, also mit welcher Tierart bezw. -Gattung wir es zu tun haben.

Ist es also unlogisch und unzweckmäßig, den Namen des ältesten oder auch nur eines alten Schriftstellers dem Tiernamen anzufügen, so ist es noch viel unlogischer und unzweckmäßiger, aus der Aufsuchung und Einführung des allerältesten, also allerunbrauchbar- 
sten Schriftstellers eine Art heiliger Pflicht der Wissenschaft zu machen und Zeit und Arbeit an solche Scheinprobleme zu vergeuden. Das ist eine der ersten Erkenntnisse, für deren allgemeine Verbreitung gesorgt werden sollte.

Das Beispiel der "Dibolia" aber zeigt uns noch weit Unlogischeres, in seiner Unzweckmäßigkeit nahezu Undenkbares.

Von den französischen Namen der Halticinengruppen, die Latreille 1825 aufstellte bezw. annahm, hat derselbe in seiner zweiten Arbeit 1829 nur die Namen Oedionychis, Psylliodes, Altica und Longitarsus (in dieser latinisierten Form) behalten. Diese Namen stimmen daher mit jenen, die Berthold durch Latinisierung der Namen aus Latreilles Erstauflage gewann, z. B. also mit den derzeit gültigen Namen Psylliodes Berth. (ex Latr.) und Longitarsus Berth. (ex Latr.) überein.

Anders liegt der Fall indes bei Dibolia. Der Name Dibolia ist nämlich eine Schöpfung lediglich der zweiten Arbeit Latreilles 1829. In der ersten Arbeit, 1825, heißt die Gruppe französisch "Altitarse“. Berthold hat 1827 diesen Namen auf "Haltitarsus" latinisiert. Der Name Haltitarsus ist nomenklatorisch gültig, er ist der erste gültige für die Gattung - di e alte Haltic in engattung Dibolia muß im Sinne der heute gültigen Nomenklaturregeln den neuen Namen "Haltitarsus Berth. (ex Latr.)“ annehmen.

Ich kann das (in den jetzigen Kriegsläuften verzeibliche) Wort nicht unterdrücken : - der Teufel soll mich holen, wenn ich diesen Unsinn mitmache. Ich möchte niemanden kränken und jedermanns Meinung achten - aber das ist ein Unsinn; es gibt kein anderes Wort dafür. Es ist ein Unsinn, nach allem, was wir soeben durchdacht haben. Es ist ein Unsinn, wenn unter dem Vorgeben, die Stabilität zu sichern, ringsum unausgesetzt fertige Stabilitäten um eines Phantoms willen zerstört werden, keine einzige Stabilität aber gesichert, d. h. für ewige Zeit unantastbar gemacht wird. Denn damit werden sich die Vertreter der absoluten Priorität, sofern sie sich über die Sachlage klar sind, wohl nicht schmeicheln, daß sie die Stabilität auch nur eines einzigen aller der Hunderttausende von Tiernamen $\mathrm{zu}$ sichern vermögen. Ebenso wie ich für die Gattung Dibolia soeben einen neuen Namen ausgegraben habe, ebenso kann heute, morgen oder in hundert Jahren für jede beliebige Tierform immer wieder ein funkelnagelneuer "allerältester" Name ausgegraben und "in seine Rechte" eingesetzt werden, alles Bestehende auf den Kopf stellend. Ich denke, das Vorhandensein der Möglichkeit, daß immer noch ein älterer 
Name exhumiert werden könne, muf uns jeder Verteidiger der absoluten Priorität zugeben und er mul zugeben, daß auf solchem Wege die Sicherung einer Stabilität völlig ausgeschlossen ist.

Unter den Händen der starren Priorität ist jeder Tiername in alle Ewigkeit an einen ängstlich dünnen Faden gehängt: jeden Augenblick kann eine ältere Beschreibung ans Tageslicht gezerrt oder irgend ein alter, die nomenklatorische Ungültigkeit des Namens bedingender Formfehler aufgedeckt werden. Unter der eisernen Faust der absoluten Priorität muß jeder alte Name ewig vor dem Gespenst des noch älteren Namens, das jede Stunde vor ihm aufstehen kann, zittern.

Ich gestehe zu, auch ich bin seinerzeit für strikte Priorität gewesen. Aber ich habe unter der Wucht der klaren Tatsachen diese Schwärmerei aufgegeben. Es könnte keine Schande für einen Forscher sein, sich durch Tatsachen von einer Meinung, die ihm auf den ersten Blick sympathisch war, abbringen zu lassen.

Doch der Name Haltitarsus ist noch in anderer Hinsicht grotesk. Nehmen wir uns die Mühe, die für seine Begründung in Betracht kommenden Autoren nachzulesen, so finden wir, daß er auf zwei unglückseligen Verwechslungen beruht.

Weder Latreille 1825, noch sein Übersetzer Berthold 1827, geben nämlich eine Beschreibung der Galerucidengruppe Altitarse-Haltitarsus. Sie sagen beide, ohne ein Wort der Charakteristik, Altitarse-Haltitarsus sei die achte „Familie“ von Illigers Gattung Haltica. Schlagen wir nun Illiger ${ }^{1}$ ) auf, so finden wir:

„b) Die hinteren Schiendorne an der Spitze zweizinkig; der Kopf ganz in den Halsschild eingesenkt:

8. Familie: Cryptocephalae.

ß) Der Hinterfuß oberhalb der Spitze der Schiene an dem oberen Ende einer rinnenförmigen schrägen Abstutzung eingelenkt (die Flügeldecken fast bei allen mit Punktstreifen).

9. Familie: Altitarses."

Wir sehen, daß Latreille, 1825, Illigers Namen „Altitarses" insofern falsch verwendete, als er ihn der achten anstatt der neunten „Familie“ Illigers beilegte. Ich sage: „falsch" verwendete, denn es ist ein Fehler und keine einfache, belanglose freihändige Vertauschung. Der Name Altitarses hängt mit der Diagnose der neunten (nicht der achten) Gruppe ursächlich zusammen, so gut wie

1) K. Illiger, Magazin f. Insektenkunde. VI, $52 ; 1807$. 
der Name "Longitarses" mit Illigers siebenter Halticinengruppe zusammenhängt. Altitarses leitet sich von altus her und bedeutet, wie dies die Diagnose der neunten Gruppe klar besagt, ein Tier, dessen Tarsen ,hoch" (d. h. oberhalb der Schienenspitze) eingelenkt sind. Diagnose und Name können nur auf die heute unter dem Namen „Psylliodes“ bekannte Halticinengattung, niemals aber auf die heutige Gattung Dibolia bezogen werden. Um Nichtigkeiten solcher Art kümmern sich allerdings die Nomenklaturgesetze ebensowenig wie um den Fehler, den Berthold bei der Transskription des Namens Altitarse ins Lateinische beging. Berthold war im Irrtum, wenn er nach der Schablone Altica =Haltica, das gleich dem Worte Longitarsus mit einem lateinischen Adjectiv (lonqus - altus) gebildete "Altitarses" in "Haltitarsus" umgoß, dadurch dessen bezeichnenden Sinn verwischte und eine Wortmißgestalt schuf.

Die starre Priorität ist schablonentreu. Sie kann die alten Gruppennamen Cryptocephalae, Altitarses usw. des geistigen Urhebers dieser klaren Gruppen, des hochverdienstlichen Illiger, nicht gelten lassen, weil die Endung nicht jene des Singulars ist (ich glaube wenigstens, dies dürfte der einzige formale Ablehnungsgrund für die Illigerschen Gruppennamen sein). Sie kann Latreilles Namen nicht anerkennen, weil sie französische statt lateinischer Endungen haben. Sie kann den Namen Dibolia, der die typische Eigenheit der Gattung wie kaum ein anderer Name treffend charakterisiert, der in aller Welt fast ein Jahrhundert lang allbekannt und stabil ist, nicht gelten lassen, weil - weil ein Name ausgegraben wurde, den niemand je kannte, den ein Übersetzer gegeben hat, dem die Materie völlig fremd war, ein Name, der auf einer Verwechslung und einem Übertragungsfehler beruht, der sachlich widersinnig und sprachlich ein Monstrum ist, der uns weder für jetzt noch für alle Zukunft irgend einen Vorteil bieten kann, den uns der altvertraute tausendfach in die Literatur geflochtene Name Dibolia nicht ebenso böte.

Diese uns widerstrebende Änderung sollen wir uns nun aufzwingen lassen, um nichts?

Kann dies im überlegten Ernste gefordert werden? Welche Vorteile erwachsen der Wissenschaft hieraus? Man denke sie durch und lege sie uns klar!

Es sind Stimmen laut geworden, die eine Festlegung endgültiger Regeln - sie seien gut oder schlecht - als die ersehnte Erlösung aus dem Chaos bedingungslos begrüßten und guthießen. Eine minder gute Regel sei besser als gar keine. Und man könnte ver- 
suchen, die unbequemen Folgen minder guter Regeln durch Spitzfindigkeiten und Schleichwege zu umgehen.

Ich lehne beides ab. Eine schlechte Regel ist schlechter als gar keine. Wo keine Regel ist, behilft sich der einfache Menschenverstand selbst und findet immerhin eine halbwegs vernunftgemäße Lösung. Eine schlechte Regel aber knebelt den gesunden Verstand.

Auf die Hintertürchen aber, durch die man vielleicht ab und zu einer allzu grotesken Folge strikter Regelbeobachtung entschlüpfen könnte, möchte ich ausdrücklich verzichten. Mit gekünstelten Kniffen wird ein gutes Recht nicht gerettet, sondern nur prostituiert.

Wo aber liegt die Lösung?

Sie liegt vor uns, wenn wir nur zwei grundsätzlich verschiedene Begriffe klar auseinanderhalten: fertige, vorhandene Stabilität und festzulegende, werdende Stabilität. Das fertig Vorhandene muß erhalten, muß geschützt - das Werdende nur muß geschaffen und für den Bau des Werdenden müssen Regeln aufgestellt werden. Eine fertige Stabilität aber ist nur dann wirklich geschützt und gesichert, wenn sie ein- für allemal allen Angriffen entzogen ist. „Kontinuität" muß an Stelle des unseligen „Priorität" das Schlagwort werden.

Wie fassen wir den Begriff „fertige Stabilität"?

Es wird den allgemeinen Anschauungen entsprechen, wenn wir einen fünfzigjährigen wissenschaftlichen Gebrauch als „fertige Stabilität" bezeichnen.

Und nun ergibt sich von selbst die Regel:

Namen, die fünfzig Jahre in wissenschaftlichem Gebrauche standen, dürfen nicht mehr aus nomenklatorischen Gründen abgeändert werden.

Dieser eiserne Schutz alles bestehenden Stabilen, dieses nachsichtslose Außerangriffstellen muß der erste Schritt zur Erreichung einer wirklichen, dauernden, sicheren - nicht einer in steter Furcht vor Gespenstern der Vergangenheit zitternden - Stabilität sein.

Über die Einzelheiten der Durchführung dieses Schutzes, über Bedenken und Einwände und ihre Widerlegung unter Berücksichtigung der Literatur soll ein an anderer Stelle erscheinender Aufsatz handeln.

Die vorliegende kleine Skizze über den Gattungsnamen Dibolia wollte vorerst nur die Frage nach der strikten Anwendung des Prioritätsprinzips ins grelle Licht der entomologischen Praxis rücken und zu kritischem Denken anregen. 


\section{$2 \mathrm{BHL}$ Biodiversity Heritage Library}

Heikertinger, Franz. 1916. "Zur Kritik der strikten Anwendung des Prioritätsprinzips in der Nomenklatur." Wiener entomologische Zeitung 35, 108-116. https://doi.org/10.5962/bhl.part.9987.

View This Item Online: https://www.biodiversitylibrary.org/item/45303

DOI: https://doi.org/10.5962/bhl.part.9987

Permalink: https://www.biodiversitylibrary.org/partpdf/9987

\section{Holding Institution}

Smithsonian Libraries

\section{Sponsored by}

Smithsonian

\section{Copyright \& Reuse}

Copyright Status: NOT_IN_COPYRIGHT

This document was created from content at the Biodiversity Heritage Library, the world's largest open access digital library for biodiversity literature and archives. Visit BHL at https://www.biodiversitylibrary.org. 